\title{
APLICACIONES DE LOS ÍNDICES DE FRAGMENTACIÓN DE LOS USOS DEL SUELO PARA CARACTERIZAR LA EXPANSIÓN URBANA
}

\author{
APPL YING LU/LC FRAGMENTATION INDICES FOR CHARACTERIZATION OF URBAN SPRAWL \\ Marta Sapena*, Luis Ángel Ruiz \\ Grupo de Cartografía GeoAmbiental y Teledetección, Departamento de Ingeniería Cartográfica, Geodesia y Fotogrametría, Universitat \\ Politècnica de València, Camí de Vera s/n, 46022, Valencia, España. marsamol@upv.es; laruiz@cgf.upv.es
}

\begin{abstract}
:
The growing number of people living in urban areas implies the need for more sustainable landscape management. The analysis and monitorization of urban areas allows for the quantification and characterization of urban sprawl processes. Currently, new geospatial and statistical databases are being developed in Europe (Urban Atlas and Urban Audit) with up-to-date and homogeneous information on urban areas, easing its comparison and monitorization, as well as the development of tools for computing urban fragmentation metrics by means of these data, such as IndiFrag. In this work, three applications of fragmentation metrics in multiple scales are presented. Firstly, an intra-urban analysis has been conducted by municipalities in Rome in two dates. Secondly, a massive analysis, comparing at the inter-urban level the fragmentation degree for one date and their growth patterns in a sample of 68 European urban areas. Finally, multiple regression models were tested to explore the prediction of socio-economic variables using the fragmentation metrics of the 68 urban areas. The outcomes show that fragmentation metrics for the analysis of morphology, composition, and urban growth, allow for quantifying urban sprawl patterns at intra- and inter-urban levels, facilitating its relation with socioeconomic variables.
\end{abstract}

Key words: Land-use/land-cover, Urban fragmentation, Fragmentation indices, IndiFrag, Urban sprawl, Socio-economic

\section{Resumen:}

El creciente aumento de población residente en áreas urbanas hace que sea necesaria una gestión del territorio más sostenible. El análisis y monitorización las áreas urbanas permite cuantificar y caracterizar los procesos de expansión urbana. Actualmente, se están desarrollando bases de datos geoespaciales y estadísticas a nivel europeo (Urban Atlas y Urban Audit) con información relativa a las áreas urbanas, de manera homogénea y actualizada, que facilitan su comparación y monitorización, además de herramientas para calcular métricas de fragmentación urbanas a partir de estos datos, como IndiFrag. En este trabajo se presentan tres aplicaciones de las métricas de fragmentación a diferentes escalas. En primer lugar, se ha elaborado un análisis intraurbano por municipios en Roma en dos fechas. En segundo lugar, un análisis masivo, donde se realiza una comparación interubana de los niveles de fragmentación, para una fecha, y de sus patrones de crecimiento, de un total de 68 áreas urbanas europeas. Por último, se han calculado modelos de predicción de variables socioeconómicas a partir de las métricas de fragmentación de las 68 áreas urbanas. Los resultados obtenidos muestran que el análisis de la morfología, composición, y del crecimiento urbano a partir de las métricas de fragmentación es adecuado para cuantificar los patrones de expansión en escalas intra e interurbanas, posibilitando su relación con variables socioeconómicas.

Palabras clave: Fragmentación urbana, Usos del suelo. Índices de fragmentación, IndiFrag, Expansión urbana, Socioeconómico

\section{Introducción}

En Europa cerca del $72 \%$ de la población vive en áreas urbanas. El continuo proceso de desarrollo de este continente altamente urbanizado, afecta más allá de las ciudades (EEA 2017). Por ello, la monitorización de áreas urbanas y periurbanas mediante la cuantificación de los cambios en los usos del suelo y sus patrones espacio-temporales, es actualmente una prioridad en la gestión de las áreas urbanas para tratar de prevenir tanto impactos medioambientales, como en la calidad de vida de la población (Malaviya et al. 2010; Wei and Zhang 2012). En los últimos años se han creado diversas iniciativas para redirigir la planificación de las áreas urbanas hacia un desarrollo más sostenible, por ejemplo: aplicando políticas de crecimiento compacto, de reciclado territorial o protección de zonas verdes, entre otros (Kompil et al. 2015).

La fragmentación del paisaje es el proceso de segregación espacial de los usos del suelo, principalmente desencadenada por actividades humanas y socioeconómicas (Wei and Zhang 2012), definido por Inostroza et al. (2013) como un patrón espacial de discontinuidad. En concreto, la fragmentación urbana refleja cómo las áreas urbanas se extienden hacia las zonas rurales (Angel et al. 2010) y su análisis temporal permite cuantificar el proceso de

"Corresponding Author: Marta Sapena, marsamol@upv.es 
expansión urbana y su eficiencia geográfica (Batty et al. 2003). Por lo tanto, cuantificar la fragmentación urbana puede ayudar en la planificación del territorio y en la toma de decisiones hacia un desarrollo urbano más sostenible (Jaeger et al. 2011).

Por ello, las métricas de fragmentación y su componente multitemporal se utilizan no sólo para describir el territorio mediante la composición espacial de los usos del suelo y sus características morfológicas, sino también para cuantificar la dinámica de los usos del suelo y el crecimiento urbano (Lausch and Herzog 2002; Malaviya et al. 2010; Sun et al. 2013).

El desarrollo de nuevas bases de datos multitemporales de usos y coberturas del suelo mediante técnicas de teledetección y SIG facilita el estudio de la fragmentación de los usos del suelo. Por ejemplo, la base de datos Europea Urban Atlas es parte del componente local del Servicio de monitorización terrestre de Copernicus (Copernicus 2010). Esta base de datos actualmente proporciona datos de 27 usos y coberturas del suelo en formato vectorial, a escala 1:10.000, de un total de 305 y 697 áreas urbanas para 2006 y 2012, respectivamente. Además, se prevé una actualización cada 6 años, siendo de gran interés para la monitorización de las áreas urbanas y periurbanas.

Por otro lado, existen diversas herramientas para analizar geoespacialmente el territorio. Un buen ejemplo es la herramienta IndiFrag, que compila un conjunto exhaustivo de índices de fragmentación aplicados para objetos cartográficos en formato vectorial (Sapena and Ruiz 2015). Al trabajar con datos de alta resolución en formato vectorial, presenta ventajas frente a otras herramientas, como el uso de las relaciones topológicas entre los objetos cartográficos, que con el formato raster no es posible. Además, al estar orientada al análisis urbano incluye métricas que otras aplicaciones orientadas a la ecología de paisaje no contemplan.

En este contexto, el objetivo de este trabajo es el de mostrar distintas aplicaciones de los índices de fragmentación y su análisis en diferentes escalas: intraurbana, interurbana y su evolución temporal, así como su relación con una selección de variables socioeconómicas relacionadas con la calidad de vida.

\section{Datos y Metodología}

En este trabajo se han utilizado dos bases de datos europeas. En primer lugar, la base datos geoespacial Urban Atlas, para obtener los datos de usos del suelo de un conjunto de áreas urbanas, para los años 2006 y 2012. Los datos proporcionados están delimitados por el área funcional urbana (functional urban area, FUA) de cada ciudad, definida como el área total de la ciudad y de los municipios colindantes en la que su población se desplaza a la ciudad por trabajo. En segundo lugar, la base de datos socioeconómicos, Urban Audit, que proporciona información estadística para un conjunto de áreas urbanas con los mismos límites administrativos que las FUAs y para varias fechas, entre ellas 2006 y 2012. El proyecto de Urban Audit representa la versión estadística del Urban Atlas (Eurostat 2016). Los institutos nacionales de estadística, de forma voluntaria, proporcionan una serie de variables relacionadas con la calidad de vida sobre demografía, vivienda, salud, transporte, etc. Al estar disponible en varias fechas, la combinación de estas bases de datos permite la comparación de áreas urbanas a lo largo del tiempo.

\subsection{Métricas de fragmentación de los usos del suelo}

Las métricas de fragmentación se han calculado con la herramienta IndiFrag, y están agrupadas en función de las propiedades que miden: área y perímetro, forma, agregación, diversidad, contraste y multitemporal. A su vez, pueden aplicarse a varios niveles jerárquicos: objeto, clase y súper-objeto (Sapena and Ruiz 2015). En este trabajo, se han calculado las métricas de los seis grupos a nivel de clase y de súper-objeto, la Tabla 1 muestra algunas de ellas. La clase es el uso del suelo de la leyenda, y el súper-objeto se refiere a los límites con los que se quiere subdividir el análisis del área urbana, este dato varía para los análisis intra e interurbano.

Las métricas de fragmentación se pueden aplicar a distintas escalas y con diferentes propósitos.

\subsubsection{Análisis de fragmentación intraurbana}

El estado de fragmentación de un área urbana puede ser analizado a través de la comparación de las métricas de fragmentación en las distintas unidades administrativas, evidenciando el comportamiento de cada clase o de su interrelación en distintas zonas de una misma área urbana.

El primer lugar, se ha realizado un análisis intraurbano, donde se estudia la fragmentación en una fecha y su evolución en una misma área urbana, comparando los valores obtenidos entre sus unidades administrativas. En este caso, se ha utilizado la FUA de Roma, donde los límites de sus municipios y los distritos del municipio de Roma forman los súper-objetos (Fig. 1).

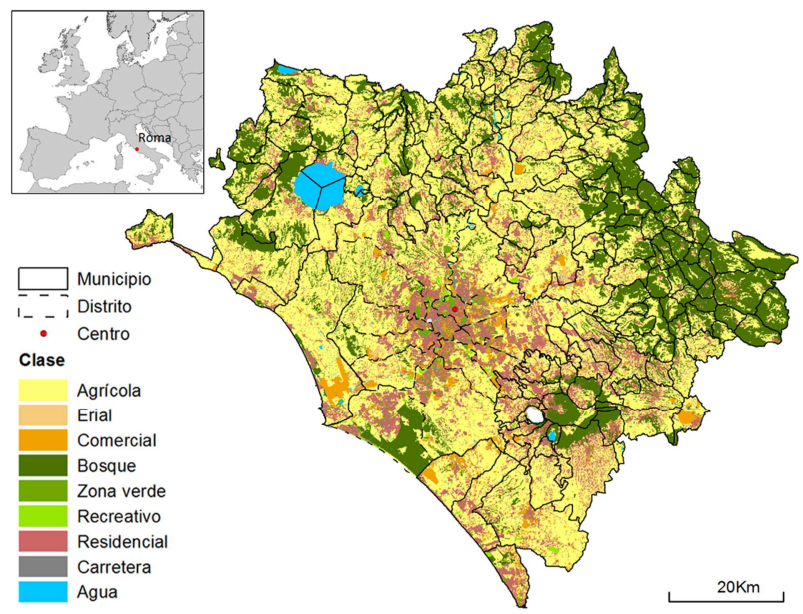

Figura 1: Zona de estudio en el análisis intraurbano. FUA de Roma con la clasificación de los usos del suelo en 2012 y unidades administrativas (municipios y distritos).

Por un lado, se han calculado las métricas a nivel de clase, para los nueve usos del suelo de la leyenda individualmente, obteniendo así un valor para cada clase y unidad administrativa, y además, las métricas a nivel de súper-objeto en 2006 y 2012 . Por otro lado, se han obtenido una serie de métricas multitemporales $y$, además, se han calculado las diferencias entre las de fragmentación en el periodo estudiado para así 
cuantificar los cambios en los usos del suelo. Finalmente, se dispone de un conjunto de valores asociados a cada unidad administrativa que permitirán la comparación entre subzonas de una misma área urbana.

Tabla 1: Descripción de la selección de métricas de fragmentación empleadas en las tres aplicaciones, la lista completa puede consultarse en Sapena and Ruiz (2015)

\begin{tabular}{|c|c|}
\hline & Nombre \\
\hline \multirow[t]{3}{*}{ Área y perímetro } & Densidad de clase (DC) \\
\hline & Tamaño medio (TM) \\
\hline & Dimensión del borde (dimB) \\
\hline \multirow[t]{3}{*}{ Forma } & Índice de forma (IF) \\
\hline & Dimensión fractal ponderada (DFP) \\
\hline & $\begin{array}{l}\text { Ratio medio del perímetro-área } \\
\text { (RMPA) }\end{array}$ \\
\hline \multirow[t]{4}{*}{ Agregación } & Número de objetos $(\mathrm{N})$ \\
\hline & $\begin{array}{l}\text { Densidad de objetos } \\
\text { (DO) }\end{array}$ \\
\hline & $\begin{array}{c}\text { Distancia estándar ponderada } \\
\text { (DEP) }\end{array}$ \\
\hline & $\begin{array}{l}\text { Distancia euclídea media del vecino } \\
\text { más próximo (DEM) }\end{array}$ \\
\hline \multirow[t]{3}{*}{ Diversidad } & Diversidad de Shannon (DSHAN) \\
\hline & $\begin{array}{l}\text { Fragmentación funcional Abs. } \\
\text { (IFFA) }\end{array}$ \\
\hline & Densidad-diversidad (DD) \\
\hline \multirow[t]{3}{*}{ Multitemporal } & $\begin{array}{l}\text { Índice de expansión medio } \\
\text { ponderado (AWM) }\end{array}$ \\
\hline & Proporción del cambio (CP) \\
\hline & Ratio de cambio (RC) \\
\hline
\end{tabular}

Descripción y unidades

Media del tamaño de los objetos de una clase o súper-objeto (m2)

Relación entre el área y perímetro de los objetos por clases

Ratio normalizado que lo compara con cuadrado de la misma área.

Media de la dimensión fractal ponderada por el área del objeto

Describe la relación entre el área y perímetro del objeto

Número de objetos por clase o súper-objeto

Número de objetos por kilómetro cuadrado de una clase o súper-objeto $(\mathrm{n} / \mathrm{km} 2)$

Distancia media de los objetos ponderada por su área al centroide del súperobjeto $(\mathrm{m})$

Distancia media entre los vecinos más próximos en una clase (m)

Abundancia proporcional de cada clase en el súper-objeto

Nivel de integración funcional de las clases

Cantidad de cada clase teniendo en cuenta su proporción total

Suma ponderada por el área de los nuevos objetos en función del tipo de crecimiento relleno, expansivo o aislado

Ratio entre la superficie cambiada y el súper-objeto (\%)

Ratio anual de cambio (\%)

\subsubsection{Análisis de fragmentación interurbana}

Para llevar a cabo el análisis interubano se han seleccionado las áreas urbanas que disponían de datos en Urban Atlas para las dos fechas y, además, que tuvieran información socioeconómica relacionada disponible en la base de datos de Urban Audit en las dos fechas. Una vez preseleccionadas las que cumplían estas condiciones, se incluye otra restricción, debido a que en el periodo de actualización del Urban Atlas (entre 2006 y 2012) la definición de FUA se revisó, cambiando los límites de algunas áreas urbanas. Por ello, en la selección final se comprobó que al menos el $80 \%$ de la superficie de las FUAs de las dos fechas fuera coincidente, para evitar así incompatibilidades con los límites de las FUAs de Urban Audit.

Finalmente, se dispone de un total de 68 FUAs europeas, localizadas principalmente en Alemania, Rumanía, Eslovaquia y Reino Unido. Del mismo modo que en el ejemplo anterior, se han aplicado las métricas a nivel de clase y súper-objeto, con diferencia de que en este caso los súper-objetos son los límites de las áreas urbanas, es decir, de la FUA. De este modo, las métricas de fragmentación permiten la comparación entre distintas áreas urbanas. En la Figura 2 se muestra la distribución de las FUAs seleccionadas y sus límites. Se puede observar la alta variabilidad de sus tamaños,

la característica común de estas FUAs es que se trata de áreas urbanas con población mayor a 100.000 habitantes.

Para reducir el número de métricas a analizar y evitar redundancias se han seleccionado aquellas que no presentan alta correlación, por un lado entre los índices de una fecha $y$, por otro, entre los multitemporales. A continuación se ha realizado un análisis de componentes principales para reducir la dimensionalidad de los datos, obteniendo así un conjunto menor de métricas significativas (Sapena et al. 2016). Este tipo de análisis estadísticos han sido aplicados en varios estudios para descartar variables muy correladas, como por ejemplo en Schwarz (2012).

\subsection{Variables socioeconómicas}

La relación de las métricas de fragmentación y variables socioeconómicas se ha realizado aprovechando la disponibilidad de la base de datos europea Urban Audit con los mismos límites administrativos que Urban Atlas. La disponibilidad de las variables en varias fechas dependerá de los países colaboradores. La combinación de las dos bases de datos multitemporales tiene un gran potencial para la monitorización de áreas urbanas. 


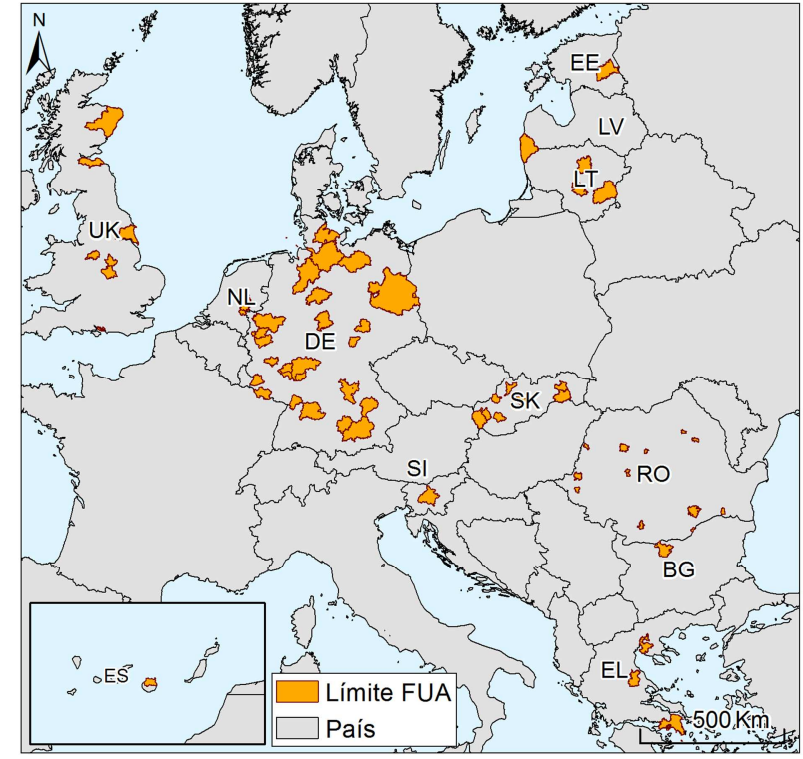

Figura 2: Zonas de estudio en el análisis interurbano con el límite de la FUA. Los países incluidos son Bulgaria (BG), Alemania (DE), Estonia (EE), Grecia (EL), España (ES),

Lituania (LT), Letonia (LV), Países Bajos (NL), Rumanía (RO), Eslovenia (SI), Eslovaquia (SK), y Reino Unido (UK). Fuente: GISCO - Eurostats (Comisión Europea).

Las métricas de fragmentación utilizadas como descriptores en el modelo son las extraídas en el análisis interurbano (sección 2.1.2). La selección de variables socioeconómicas (variables dependientes) está restringida para que todas tengan dato en al menos 25 de las 68 FUAs y así tener suficientes observables en el análisis estadístico. En este caso, se muestra un total de 10 variables para 2006, 2012 y sus diferencias, con las que se obtuvieron relaciones significativas con las métricas (Tabla 2).

Tabla 2: Listado, descripción y número de observables de las variables socioeconómicas seleccionadas

\begin{tabular}{|c|c|c|}
\hline Variable & Descripción & FUAs \\
\hline pop1 & Población 1 enero & 65 \\
\hline popres & $\begin{array}{l}\text { Densidad de población en suelo } \\
\text { residencial }\end{array}$ & 65 \\
\hline inc & $\begin{array}{c}\text { Media del ingresos disponibles anuales } \\
\text { por hogar }\end{array}$ & 25 \\
\hline prchild & Proporción de hogares con menores & 30 \\
\hline act & Población activa económicamente & 54 \\
\hline rtact & Ratio de actividad & 25 \\
\hline unemp & Población sin empleo & 46 \\
\hline rtun & Ratio de desempleo & 30 \\
\hline avlen & $\begin{array}{l}\text { Longitud media de camino al trabajo en } \\
\text { coche }\end{array}$ & 25 \\
\hline killac & $\begin{array}{l}\text { Personas fallecidas en accidente de } \\
\text { coche cada } 10.000 \text { habitantes }\end{array}$ & 48 \\
\hline
\end{tabular}

Se ha realizado un análisis exploratorio de predicción de las variables socioeconómicas basado en métricas de fragmentación y sus cambios, mediante el método estadístico de regresión múltiple paso a paso. Los modelos de regresión se han calculado para 2006, 2012 y sus diferencias.

\section{Resultados y discusión}

En esta sección se comentan los resultados obtenidos en los tres análisis, se ha dividido por las mismas subsecciones que en el apartado de metodología.

\subsection{Interpretación espacio-temporal intraurbana}

La propiedad de agregación muestra la tendencia de los elementos a agregarse espacialmente. Un buen ejemplo es la Distancia euclídea media del vecino más próximo (DEM), que informa sobre la separación entre los elementos de una misma clase. Este índice para la clase residencial en Roma (Fig. 3) muestra dos comportamientos diferenciados. Se observan aquellos municipios con un grado de concentración de los elementos de uso residencial muy elevado en color azul, con una distancia media entre ellos menor o igual a 30 metros, sugiriendo que se trata de áreas muy compactas en cuanto a este uso. Esta información puede completarse con símbolo gradual que representa la Densidad de la clase (DC) residencial en ese municipio. El análisis combinado de estas variables facilita su interpretación. Se puede observar que los municipios con baja densidad residencial en su mayoría presentan distancias medias mayores a los 85 metros, indicando en este caso que los municipios de la zona norte y nordeste de Roma presentan una cobertura residencial poco densa y muy fragmentada con respecto al resto de municipios en 2012.

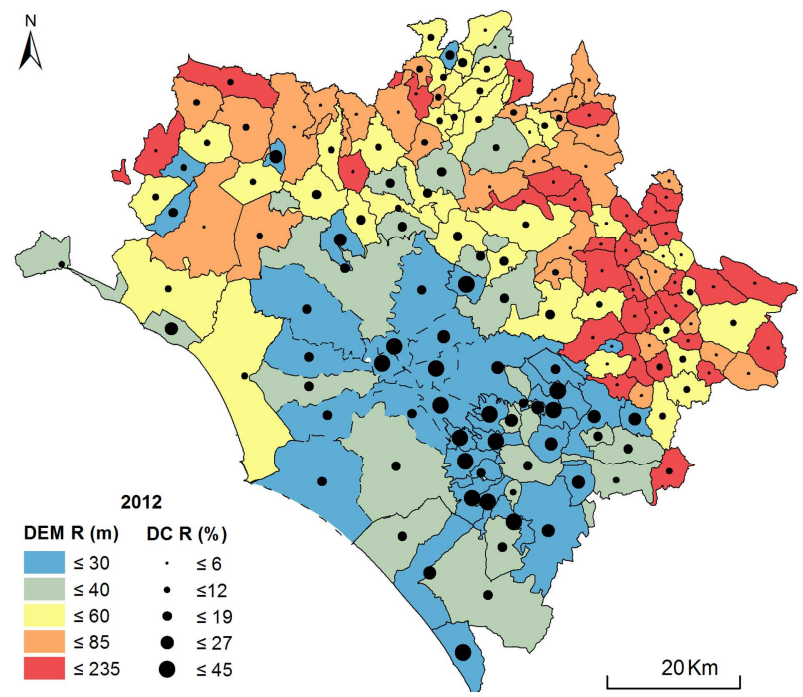

Figura 3: Representación gráfica de la Distancia Euclídea Media del vecino más próximo (DEM) y de la Densidad de Clase (DC) del uso residencial para el año 2012.

Por otro lado, la diversidad es una propiedad que informa sobre la riqueza, en este caso en función del número de clases presente, y su uniformidad, el equilibrio entre ellas, en una unidad administrativa. Esta propiedad puede representarse a partir del índice Densidad-Diversidad (DD) dotando a cada municipio de un valor entre 0 (cuando sólo hay una clase) y 9 (igual al número de clases, cuando están todas presentes en la misma proporción), por ejemplo, en la Figura 4 se 
observa cómo los valores más bajos se encuentran en municipios principalmente de uso forestal o agrícola.

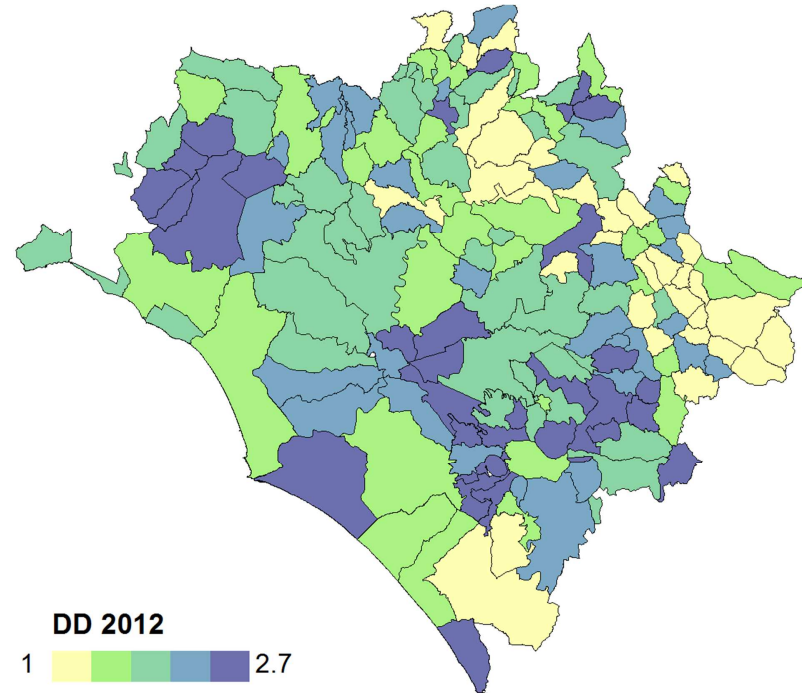

Figura 4: Representación gráfica de la Densidad-Diversidad (DD) para el año 2012.

Cuando se analizan las métricas de fragmentación a lo largo del tiempo, se pueden obtener patrones de crecimiento de los municipios. Por ejemplo, la combinación de los índices de Expansión media ponderada (AWM) y la Proporción del cambio (CP) refleja la tendencia de crecimiento más o menos fragmentado por municipios de una misma área urbana. Un ejemplo de su interpretación puede verse en (Sapena et al. 2015). Otro ejemplo combinando métricas se presenta en la Figura 5 , donde se muestra la cantidad y tipo de crecimiento de la clase residencial por municipio y, a su vez, la proporción de cambio que ha tenido lugar en la clase de zonas verdes. Los tres tipos de crecimiento descritos son: relleno, cuando el nuevo objeto esta parcial o totalmente rodeado por otro de la misma clase; expansivo, cuando se desarrolla en la frontera de la clase; y aislado, si está totalmente separado de la clase (Wilson et al. 2003). La combinación del crecimiento de tipo relleno y expansivo indica un crecimiento compacto, mientras que el tipo aislado es un crecimiento fragmentado. Se observa de nuevo en la zona norte y nordeste, que además de ser municipios muy fragmentados en 2012, el proceso de expansión entre 2006 y 2012 fue en su mayoría muy disperso. Al contrario, los municipios costeros y de la zona sur presentan un crecimiento residencial compacto. Por otro lado, el crecimiento de las zonas verdes en la parte periférica de la FUA es prácticamente nulo, mientras que en los distritos de Roma hay un aumento general de hasta el 0,8\%, excepto en los distritos más céntricos por ser ya muy compactos.

En resumen, se han observado dos zonas claramente diferenciadas a partir de las métricas de fragmentación aplicadas de manera intraurbana en la FUA de Roma. En primer lugar, la zona periférica de la FUA presenta un paisaje menos denso y diverso, y más fragmentado, donde su proceso de expansión sigue esa tendencia y las zonas verdes no han aumentado, probablemente al tratarse de zonas forestales con edificaciones aisladas y mucha vegetación. En segundo lugar, la zona costera y central de la FUA, presenta mayor diversidad (ya que hay más variedad en los usos de suelo urbanos, que son los que principalmente forman la leyenda), tienen mayor densidad de suelo residencial y su distribución espacial es más compacta, acompañado de una tendencia de crecimiento de la clase residencial muy agregada, y en general, un aumento positivo de las zonas verdes.

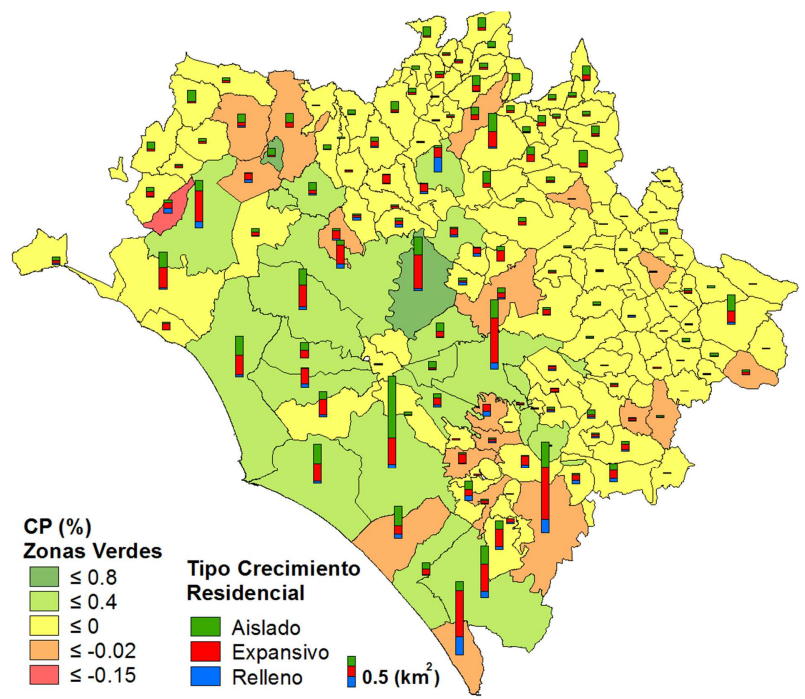

Figura 5: Representación gráfica de la proporción del cambio (CP) de la clase de zonas verdes y el tipo de crecimiento de la clase residencial entre 2006 y 2012, por municipio.

\subsection{Comparación interurbana}

Se ha llevado a cabo un cálculo masivo de métricas de fragmentación en 68 áreas urbanas, que permite realizar comparaciones del estado de fragmentación en un momento concreto y de su evolución. Se han realizado varios análisis, por ejemplo, en la Figura 6 se representan las 68 FUAs clasificadas por países y ponderadas por su tamaño, donde su posición en el eje de abscisas muestra las distancias medias del vecino más próximo del uso residencial, y el eje de las ordenadas su densidad en 2012. Las FUAs localizadas en el extremo derecho presentan un grado de fragmentación mucho más elevado que el resto, además, se caracterizan por su baja densidad de suelo residencial. Sin embargo, las que están en el extremo izquierdo, independientemente de su densidad, son áreas muy compactas en cuanto a este uso.

La Figura 7 muestra cinco ejemplos extraídos de la Figura 6, con distintos niveles de fragmentación, densidad y tamaño de FUA. Se observa que las FUAs más fragmentadas (Aberdeen en Reino Unido y Liepaja en Letonia) tienen un núcleo residencial localizado en la ciudad, mientras que el resto de suelo residencial se encuentra disperso en toda su extensión. Al contrario, en las más compactas, la distribución del uso residencial es más homogénea espacialmente, tanto en la ciudad como en el resto de municipios.

En general, se observa que las FUAs alemanas y eslovacas están agrupadas en la Figura 6, indicando en este caso que la clase residencial en 2012 es muy compacta. Al contrario, las FUAs de Reino Unido tienen un comportamiento más variado, ya que presentan valores de DEM muy dispares, como por ejemplo con Aberdeen y Portsmouth, en la Figura 7. 


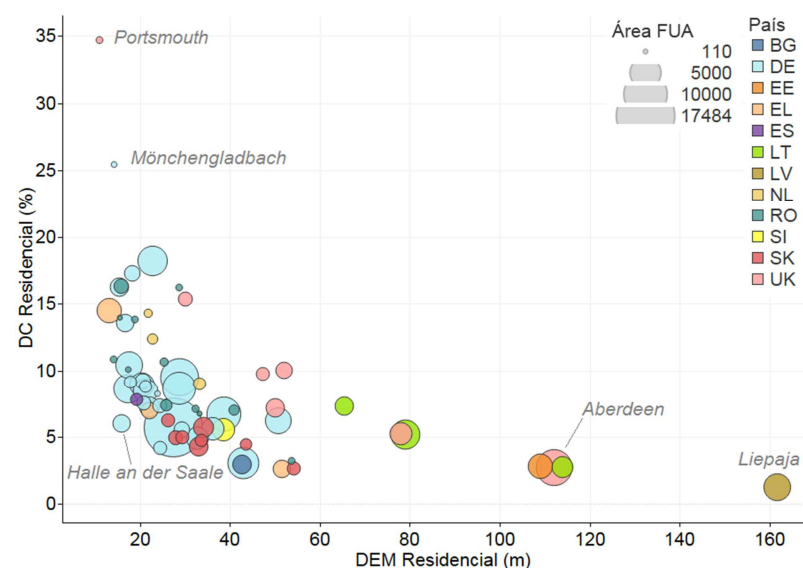

Figura 6: Distribución de las FUAs en función de la Distancia euclídea media del vecino más próximo (DEM) y de la Densidad de clase del uso residencial. El tamaño del punto viene definido por el tamaño de la FUA y el color por el país al que pertenece.

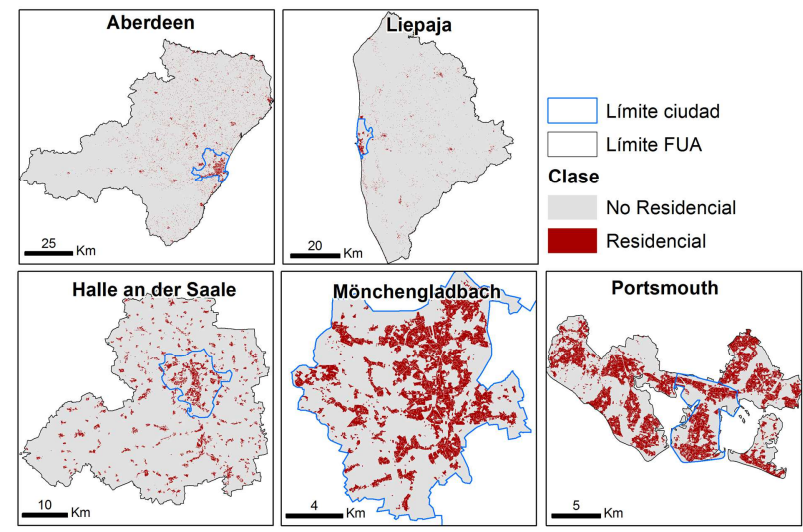

Figura 7: Ejemplo de las FUAs con mayor y menor grado de fragmentación respecto al uso residencial en 2012, puede verse su localización en la Figura 6 mediante una etiqueta con su nombre.

Sin embargo, el análisis temporal, independientemente del estado actual de fragmentación, informa sobre el proceso de expansión urbana que está teniendo lugar en una determinada zona. En la Figura 8 se muestran las FUAs en función de la Proporción de cambio (CP) de la clase residencial en el eje de ordenadas y el Índice de Expansión medio ponderado (AWM) de la misma clase en las abscisas. El tamaño del punto está en función de la Densidad de la clase (DC) residencial que resulta en 2012, y el color indica el país al que pertenece. En primera instancia, se observa que las FUAs rumanas son las que más se han desarrollado, con un crecimiento mixto entre fragmentado y compacto, alcanzando unas densidades de suelo residencial de alrededor del $15 \%$. En segundo lugar, se observan tendencias en los procesos de expansión en función del país, puede observarse en la Figura 8 cómo las FUAs se encuentran agrupadas por países. Por ejemplo, las FUAs alemanas de una densidad intermedia-alta en 2012, han experimentado un crecimiento de la clase residencial reducido, en su mayoría con una tendencia compacta, de manera similar sucede con las de Reino Unido. Eslovaquia, también tiene un patrón definido, con un crecimiento también suave, pero en este caso más fragmentado. Por último, el proceso de crecimiento más fragmentado se encuentra en la FUA española (Las
Palmas de Gran Canaria), ya que el tipo de crecimiento aislado es un $68 \%$ del crecimiento residencial total, sin embargo, en 2012 se considera una de las FUAs más compactas a pesar de que su proceso de expansión haya sido mayormente disperso.

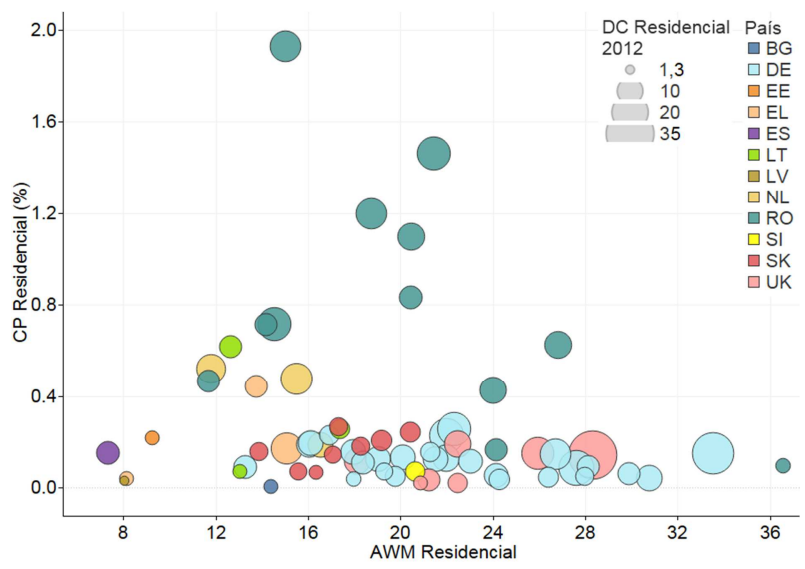

Figura 8: Distribución de las FUAs en función de la Proporción del Cambio Residencial (CP) y de su Índice de Expansión Medio Ponderado (AWM). El tamaño del punto viene definido por la Densidad de la Clase residencial en el año 2012 y el color por el país al que pertenece.

\subsection{Modelos de predicción socioeconómicos}

Una tercera aplicación de las métricas de fragmentación es la de su relación con las variables socioeconómicas. Por lo tanto, se ha realizado un análisis exploratorio en el que se han calculado varios modelos para evaluar las relaciones existentes entre las métricas de fragmentación y las variables socioeconómicas. En la Tabla 3 se muestran los resultados de algunos modelos con coeficiente de determinación mayor de 0,5. El número de observaciones varía en cada modelo en función de los datos disponibles, el significado de cada variable y número de FUAs u observables se puede consultar en las Tablas 1 y 2.

En general, se observa que los modelos de población (pop1) están muy influenciados por las métricas que dependen del tamaño, por ejemplo, número de objetos (N) y tamaño medio (TM) del uso residencial. Sin embargo, cuando se omiten del modelo también se obtienen modelos en función de la diversidad $\mathrm{y}$ distribución de los objetos con un $\mathrm{R}^{2} \approx 0,75$. Por otro lado, la variable de densidad de población respecto al uso residencial (popres) no está tan afectada por el tamaño, pero sí por la forma y su configuración. En cuanto a la variable relacionada con los ingresos medios (inc), sus observables se reducen a las FUAs alemanas, aunque no sea un modelo muy robusto, está influido por la heterogeneidad, tamaño de los objetos y densidad de zonas verdes. Mientras que su variación en el tiempo, se puede explicar por la variación del tamaño de los elementos de uso recreativo y el cambio de la clase comercial. Por otro lado, las variables relacionadas con el empleo en una fecha (act y unemp) están relacionadas en gran medida con el uso residencial y comercial, en concreto su tamaño, superficie y distribución. 
Tabla 3: Listado de los modelos de regresión con las variables socioeconómicas como dependientes y las de fragmentación independientes. La letra tras el índice indica la clase, donde residencial $(R)$, comercial $(C)$, recreativo $(L)$ y zonas verdes

(G). Si la variable es negativa significa que tiene una influencia negativa en el modelo. La "d" significa la diferencia de la métrica entre las dos fechas.

\begin{tabular}{|c|c|c|c|}
\hline $\begin{array}{l}\text { Var. Socio } \\
\text { económica }\end{array}$ & Fecha & Variables descriptoras & $\begin{array}{c}R^{2} \\
\text { ajust. }\end{array}$ \\
\hline \multirow[t]{2}{*}{ pop1 } & 2006 & DSHAN, N_R, TM_R & 0,90 \\
\hline & 2012 & RMPA, -DEM_C, TM_R, N_R & 0,93 \\
\hline \multirow[t]{2}{*}{ popres } & 2006 & RMPA, TM_C, -DEM_C, -IF_L & 0,80 \\
\hline & 2012 & RMPA, IFFA, -DEM_C & 0,56 \\
\hline inc & $06 / 12$ & -dTM_L, RC_C & 0,65 \\
\hline \multirow[t]{2}{*}{ prchild } & 2006 & -TM, DEM_L & 0,79 \\
\hline & 2012 & RMPA, TM_L, DEM_L & 0,62 \\
\hline act & 2006 & DEP, -DEM_C, N_R, TM_R & 0,92 \\
\hline rtact & $06 / 12$ & dDEM_G, dDimB_L & 0,75 \\
\hline unemp & 2006 & TM_C, N_R & 0,72 \\
\hline rtunemp & $06 / 12$ & -dDFP_G, RC_R & 0,53 \\
\hline avlen & $06 / 12$ & $\underset{\text { RC_C }}{\text { dDimB_C, dRMPA_L, dDEP_R, }}$ & 0,82 \\
\hline killac & 2006 & IFFA, IF_G, DEM_L, -DD_R & 0,66 \\
\hline
\end{tabular}

Los modelos calculados para los ratios de actividad y desempleo (rtact y rtun) revelan una relación positiva con el crecimiento de zonas verdes y cambios en los servicios de recreo. Por último, las variables relacionadas con el transporte (avlen), tienden a cambiar en función de los cambios en las distancias al centro del uso residencial y la intensidad de cambio del uso comercial.

Aunque se han observado relaciones entre las métricas de fragmentación y las variables socioeconómicas, hay otras variables que todavía no han podido analizarse por no encontrar ninguna correlación significativa con las métricas, como por ejemplo variables relacionadas con la fertilidad, mortalidad, nivel de educación, etc.
Además, la falta de disponibilidad de datos en el periodo estudiado hace que el análisis esté limitado, sin embargo, la base de datos estadísticos está todavía en proceso de desarrollo y se espera, además de su actualización, nuevas variables relacionadas con la calidad de vida de la población.

\section{Conclusiones}

Este trabajo muestra el potencial en cuanto a aplicaciones e interpretación de las métricas de fragmentación en entornos urbanos, en concreto para la monitorización de la expansión urbana. Los SIG permiten el análisis espacial de los datos y el desarrollo de aplicaciones como IndiFrag, que hacen posible la creación de metodologías, que permiten cuantificar y evaluar la evolución del paisaje urbano.

Las tres aplicaciones presentadas evidencian la aptitud de las métricas de fragmentación de los usos del suelo para describir la morfología, estructura y evolución de áreas urbanas a distintas escalas, facilitando así la comparación inter e intraurbana de los procesos de expansión urbana, incluso en periodos cortos de tiempo. También se ha podido comprobar la relación que existe entre las variables socioeconómicas y la distribución espacial de los usos del suelo, su complejidad y los cambios estructurales de las áreas urbanas. Este estudio podría ayudar al desarrollo de nuevas políticas sostenibles a nivel local, ya que las áreas urbanas crecen en función de las demandas económicas y de la población, y este trabajo evidencia su interrelación. Sin embargo, todavía quedan aspectos a analizar a causa de la falta de datos del Urban Audit. En trabajos futuros se pretende evaluar el comportamiento de las métricas de fragmentación frente a un conjunto de escenarios modelizados que simulen patrones de crecimiento diferentes.

\section{Agradecimientos}

Este trabajo ha sido financiado por el Ministerio de Economía y Competitividad y FEDER, en el marco del proyecto CGL2016-80705-R y con el contrato del Fondo de Garantía Juvenil PEJ-2014-A-45358.

\section{References}

ANGEL, S., PARENT, J. and CIVCO, D.L., 2010. The fragmentation of urban footprints: global evidence of sprawl, 19902000. Lincoln Institute of Land Policy, Working Paper, pp. 1-100.

BATTY, M., BESUSSI, E. and CHIN, N., 2003. Traffic, urban growth and suburban sprawl. Centre for Advanced Spatial Analysis, Working Paper 70, London, UK, pp. 1-12.

COPERNICUS, 2010. Copernicus Land Monitoring Services - The European Earth Observation Programme - Local Component. Disponible: http://land.copernicus.eu/local [7/28,2015].

EEA, 2017. European Environmental Agency - About urban environment. Disponible: https://www.eea.europa.eu/themes/sustainability-transitions/urban-environment/about-urban-environment $[5 / 01,2017]$.

EUROSTAT, 2016. Cities (Urban Audit) - Database. Disponible: http://ec.europa.eu/eurostat/web/cities/data/database $[3 / 15,2016]$

INOSTROZA, L., BAUR, R. y CSAPLOVICS, E., 2013. Urban sprawl and fragmentation in Latin America: A dynamic quantification and characterization of spatial patterns. Journal of Environmental Management, 115, pp. 87-97. DOI:10.1016/j.jenvman.2012.11.007.

JAEGER, A.G., SOUKUP, T., MADRIÑÁN, L.F., SCHWICK, C. and KIENAST, F., 2011. Landscape fragmentation in Europe, EEA Report. DOI:10.2800/78322. 
KOMPIL, M., AURAMBOUT, J.-P., BARRANCO, R.R., BARBOSA, A., CRISIONI, C.J.-, PISONI, E., ZULIAN, G., VANDECASTEELE, I., TROMBETTI, M., VIZCAINO, P., VALLECILLO, S., SILVA, F.B. E, BARANZELLI, C., RIVERO, I.M., MAES, J. and LAVALLE, C., 2015. European cities: territorial analysis of characteristics and trends An application of the LUISA Modelling Platform (EU Reference Scenario 2013 - Updated Configuration 2014),EUR 27709 EN. DOI:10.2788/737963.

LAUSCH, A. and HERZOG, F., 2002. Applicability of landscape metrics for the monitoring of landscape change: Issues of scale, resolution and interpretability. Ecological Indicators, 2, pp. 3-15. DOI:10.1016/S1470-160X(02)00053-5.

MALAVIYA, S., MUNSI, M., OINAM, G. and JOSHI, P.K., 2010. Landscape approach for quantifying land use land cover change (1972-2006) and habitat diversity in a mining area in Central India (Bokaro, Jharkhand). Environmental Monitoring and Assessment, 170, pp. 215-229. DOI:10.1007/s10661-009-1227-8.

SAPENA, M. and RUIZ, L.A., 2015. Descripción y cálculo de índices de fragmentación urbana: Herramienta IndiFrag. Revista de Teledección, 43, pp. 77-89. DOI:http://dx.doi.org/10.4995/raet.2015.3476.

SAPENA, M., RUIZ, L.A. and GOERLICH, F.J., 2016. Analysing relationships between urban land use fragmentation metrics and socio-economic variables. The International Archives of the Photogrammetry, Remote Sensing and Spatial Information Sciences, XLI-B8, pp. 1029-1036. DOI:10.5194/isprsarchives-XLI-B8-1029-2016.

SAPENA, M., RUIZ, L.A. and PALOMAR, J.M., 2015. Estudio evolutivo de los usos del suelo urbano mediante índices de distribución espacio-temporal. XVI Congreso de la Asociación Española de Teledetección. Sevilla. pp. 64-67.

SCHWARZ, N., 2010. Urban form revisited-selecting indicators for characterising European cities. Landscape and Urban Planning, 96, pp. 29-47. DOI:10.1016/j.landurbplan.2010.01.007.

SUN, C., WU, Z.F., LV, Z.Q., YAO, N. and WEI, J.B., 2013. Quantifying different types of urban growth and the change dynamic in Guangzhou using multi-temporal remote sensing data. International Journal of Applied Earth Observation and Geoinformation, 21, pp. 409-417. DOI:10.1016/j.jag.2011.12.012.

WEI, Y. and ZHANG, Z., 2012. Assessing the fragmentation of construction land in urban areas: An index method and case study in Shunde, China. Land use policy, 29, pp. 417-428. DOI:10.1016/j.landusepol.2011.08.006.

WILSON, E.H., HURD, J.D., CIVCO, D.L., PRISLOE, M.P. and ARNOLD, C., 2003. Development of a geospatial model to quantify, describe and map urban growth. Remote Sensing of Environment, 86, pp. 275-285. DOI:10.1016/S00344257(03)00074-9. 